\title{
Screening of genomic imbalances in glioblastoma multiforme using high-resolution comparative genomic hybridization
}

\author{
VLADIMÍRA VRANOVÁ ${ }^{1,5}$, EVA NECESALOVÁ2,5, PETR KUGLÍK ${ }^{1}$, PAVEL CEJPEK ${ }^{3}$, \\ MARTINA PESÁKOVÁ ${ }^{5}$, EVA BUDÍNSKÁ ${ }^{4}$, JIRINA RELICHOVÁ ${ }^{1}$ and RENATA VESELSKÁ ${ }^{1}$ \\ ${ }^{1}$ Department of Genetics and Molecular Biology, Institute of Experimental Biology, Faculty of Science; \\ Departments of ${ }^{2}$ Biology and ${ }^{3}$ Neurosurgery, Faculty of Medicine; ${ }^{4}$ Centre of Biostatistics and \\ Analyses, Faculty of Medicine and Faculty of Science, Masaryk University; ${ }^{5}$ Department of \\ Medical Genetics, University Hospital, Brno, Czech Republic
}

Received October 4, 2006; Accepted November 8, 2006

\begin{abstract}
Comparative genomic hybridization (CGH) is a molecular cytogenetic technique that allows the genomewide analysis of DNA sequence copy number differences. We applied conventional $\mathrm{CGH}$ and the recently developed high-resolution $\mathrm{CGH}$ (HR-CGH) to tumour samples from 18 patients with glioblastoma multiforme (GBM) in order to compare the sensitivity of $\mathrm{CGH}$ and HR-CGH in the screening of chromosomal abnormalities. The abnormalities were studied in topologically different central and peripheral tumour parts. A total of 78 different changes were observed using $\mathrm{CGH}$ (0-16 per tumour, median 3.5) and 154 using HR-CGH (0-21 per tumour, median 6). Using HR-CGH, losses were more frequent than gains. The representation of the most prominent changes revealed by both methods was similar and was comprised of the amplification of $7 q 12$ and $12 q 13-q 15$, the gain of $7,3 q$ and 19 , and the loss of $10,9 p$, and $13 q$. However, HR-CGH detected certain other abnormalities (the loss of $6,14 q, 15 q$ and $18 q$, and the gain of 19), which were rarely revealed by $\mathrm{CGH}$. Using $\mathrm{HR}-\mathrm{CGH}$, the numbers and types of chromosomal changes detected in the central and peripheral parts of GBM were almost the same. The loss of chromosomes 10 and $9 \mathrm{p}$ and the gain of chromosomes 7
\end{abstract}

Correspondence to: Dr Renata Veselska, Laboratory of Tumor Biology and Genetics, Department of Genetics and Molecular Biology, Institute of Experimental Biology, Faculty of Science, Masaryk University, Kotlarska 2, 61137 Brno, Czech Republic E-mail: veselska@sci.muni.cz

Abbreviations: CGH, comparative genomic hybridization; DAPI, 4,6-diamidino-2-phenylindole; EGFR, epidermal growth factor receptor; GBM, glioblastoma multiforme; HR-CGH, high-resolution comparative genomic hybridization; I-FISH, interphase-fluorescence in situ hybridization; TSG, tumour suppressor gene

Key words: comparative genomic hybridization, high-resolution comparative genomic hybridization, glioblastoma multiforme, molecular cytogenetics and 19 were the most frequent chromosomal alterations in both tumour parts. Our results from the GBM analysis show that HR-CGH technology can reveal new, recurrent genetic alterations involving the genes known to participate in tumorigenesis and in the progression of several human malignancies, thus allowing for a more accurate genetic characterization of these tumours.

\section{Introduction}

Glioblastoma multiforme (GBM) (WHO grade IV) is the most malignant type of brain tumour of astrocytic origin (1). GBM is usually divided into two groups: (i) Primary or de novo GBM is clinically aggressive, occurs in older patients and is characterized by a more complex karyotype, (ii) secondary GBM, developed from pre-existing low-grade astrocytomas, occurs in younger patients and has a better prognosis (2). The tumorigenesis of GBM is the consequence of the accumulation of genetic events that confer a growth advantage to a glial cell. Genetic alterations occur at multiple sites, and the most frequent of these include the loss of chromosomes 10 and $9 p$ and the gain of the entire chromosome 7 with a 7p12 (EGFR) amplification $(3,4)$. Different chromosomal aberrations and their combinations can provide useful information with respect to tumour classification $(5,6)$, prognosis $(7,8)$ and response to therapy $(9,10)$.

Various improvements in molecular cytogenetic techniques allow for a more precise determination of chromosomal changes. Chromosome comparative genomic hybridization $(\mathrm{CGH})$ represents the possibility of obtaining genome-wide analyses of DNA sequence copy number differences (11) and has been successfully applied to GMB studies $(12,13)$. This method detects changes that are present in $\geq 50 \%$ of the specimen cells, with the affected region exceeding 5-10 Mb. However, many chromosomal aberrations are present in an abundance lower than $50 \%$ and this may result in problems with their identification. High-resolution CGH (HR-CGH), developed by Kirchhoff et al (14), is primarily suited for the detection and localization of genomic changes down to 3-5 Mb and/or those with lower aberrant clone prevalence (15). HRCGH was successfully used in clinical genetic studies to reveal 
subtle chromosomal rearrangements in dysmorphic and mentally retarded individuals with normal karyotypes $(16,17)$ as well as for the diagnostic and prognostic classification of haematological malignancies (18).

As with previously published methods (14), we developed our own HR-CGH modifications using specialized software from Laboratory Imaging, Ltd. (Prague, Czech Republic). Subsequently, HR-CGH together with conventional CGH was employed in the analysis of samples from 18 patients with GBM in order to compare the sensitivity of these two methods. HR-CGH was very effective in the detection of chromosomal changes. Compared with CGH, HR-CGH was able to reveal nearly $50 \%$ more aberrations. Because GBM is characterized not only by intertumoral but also by intratumoral heterogeneity in both histomorphology and genetic changes (19-21), we also compared the incidence of various chromosomal changes in both the peripheral and central parts of tumours. Nevertheless, our results showed that these two sections were not genetically different.

\section{Materials and methods}

Tumour material. The samples of GBM from 18 adult patients (12 male/6 female; median age, 65.5 years; range, 28-76 years) were obtained from the Department of Neurosurgery, University Hospital in Brno. All the samples were histologically characterized according to the WHO classification (1) as astrocytoma grade III and IV. The central part of the tumour came from the geometric hub of the necrotic and cystic tumour tissue and the peripheral part was taken from the line dividing the pathological and healthy tissue. The tumour samples were frozen in liquid nitrogen immediately after resection and were stored at $-70^{\circ} \mathrm{C}$. Portions of both sections of each tumour were used for tumour touch imprints. Briefly, a slice of the tumour was removed, and the surface created by the cutting was printed on a microscopic slide. The slides were then dried and fixed in Carnoy's fixative. These tumour touch imprints were utilized for the interphasefluorescence in situ hybridization (I-FISH) examination. The DNA was extracted from the remaining frozen tissue using standard chloroform extraction procedures and examined by $\mathrm{CGH} / \mathrm{HR}-\mathrm{CGH}$.

CGH and HR-CGH protocols. Phytohemagglutinin-stimulated normal lymphocytes from karyotypically normal males were prepared as the metaphase targets for the $\mathrm{CGH} / \mathrm{HR}-\mathrm{CGH}$ experiments using standard protocols. CGH was performed according to the manufacturer's protocol (Abbott-Vysis, Inc., Downers Grove, IL, USA). Briefly, 2 ng tumour DNA, extracted from native frozen tissue using chloroform extraction, was labelled with Spectrum Green-dUTP (AbbottVysis) by nick translation. Simultaneously, normal human genomic DNA from peripheral blood lymphocytes was labelled with Spectrum Red-dUTP (Abbott-Vysis). The test and reference DNA were then ethanol-precipitated in the presence of $15 \mu \mathrm{g}$ human Cot-1 DNA (Abbott-Vysis) and dissolved in $10 \mu 1$ hybridization buffer (50\% formamide/10\% dextran sulfate/2x SSC, $\mathrm{pH} 7.0$ ). The probe mixture was denatured and hybridized to denatured normal metaphase preparations at $37^{\circ} \mathrm{C}$ in a humidified chamber for $48-72 \mathrm{~h}$.
After hybridization, the slides were washed in $0.5 \mathrm{x}$ SSC at $74^{\circ} \mathrm{C}$ and $2 \mathrm{x}$ SSC at room temperature and then counterstained with 4,6-diamidino-2-phenylindole (DAPI) solution.

Representative images of at least 10 metaphases per case were captured using an Olympus BX61 microscope and a CCD Camera COHU 4910. The chromosomes were karyotyped and ratio profiles were calculated using the software program, LUCIA G 4.82 - KARYO/FISH/CGH/CGHAdvanced Statistics (Laboratory Imaging).

For the CGH analysis, the losses and gains of the chromosomal regions were detected when the green:red ratio profiles deviated $20 \%$ from a ratio of $1(<0.80$, losses; $>1.20$, gains $)$. Amplification was implied in the profiles with sharp rising peaks relative to the rest of the chromosomal profiles. The centromeres and acrocentric p-arms were excluded from the CGH analysis.

As mentioned above, HR-CGH was developed according to the published methods (14). In our study, the $99.5 \%$ dynamic standard reference interval was based on an average of 17 CGH analyses from healthy donors with normal karyotypes. The interval for each chromosome was then analyzed by a special software program, LUCIA G 4.82 Advanced Statistics (Laboratory Imaging). Chromosomal abnormalities were detected by comparing this $99.5 \%$ dynamic standard reference interval to the $99.5 \%$ confidence interval of the mean ratio profile of the test samples. Regions where the two sets of intervals did not overlap were considered aberrant. The centromeres, telomeres and acrocentric p-arms were excluded from the HR-CGH analysis.

For system sensitivity and specificity tests, patients with microdeletion syndromes (Prader-Willi/Angelman and DiGeorge) were analyzed and the sensitivity of the system was established at an interval of 4-5 Mb. To determine clone prevalence that can be identified by HR-CGH, particular chromosome aberrations detected by HR-CGH were verified by I-FISH with specific probes on the tumour touch imprints as described below.

(I-FISH). To verify the HR-CGH results, I-FISH was performed on the respective tumour touch imprints. Slides with tumour imprints were fixed in Carnoy's fixative, codenatured and allowed to hybridize with commercially available centromeric probes from Aquarius ${ }^{\circledR}$, Cytocell, UK [Enumeration Probe Chromosome 10 (Red)], and [Enumeration Probe Chromosome 7 (Green)], locus-specific probes from Q-BIOgene, France [17p13 specific probe (Green)/17 $\alpha$ Satellite Probe (Red)], and centromeric or locus-specific probes from Abbott-Vysis [LSI 1p36 SO/1q25 SG Probe, LSI ALK (2p23) Dual Color Probe, LSI IgH SG/ MYC SO/CEP 8 SA Probe, Her2/Neu17q11.2-12 SO/CEP 17 SG, LSI 19q13 SO/19p13 SG Probe, and LSI abl SO/bcr SG] according to the manufacturer's instructions. After posthybridization washes, the slides were counterstained with DAPI and evaluated using an Olympus BX61 fluorescence microscope. At least 100 nuclei were captured and evaluated using a CCD camera and LUCIA software as described above.

Statistical analysis. The spectrum and frequency of the chromosomal aberrations were compared between CGH and HR-CGH as well as between the central and peripheral tumour 
Table I. The number of DNA copy number changes in the tumour samples from 18 patients with GBM detected by CGH and HR-CGH.

\begin{tabular}{|c|c|c|c|c|c|c|c|c|}
\hline \multirow[b]{2}{*}{ Patient } & \multirow[b]{2}{*}{ Sex } & \multirow[b]{2}{*}{ Age } & \multicolumn{3}{|c|}{ CGH } & \multicolumn{3}{|c|}{ HR-CGH } \\
\hline & & & $\mathrm{G}$ & $\mathrm{L}$ & $\mathrm{T}$ & G & $\mathrm{L}$ & $\mathrm{T}$ \\
\hline 1 & M & 65 & 0 & 0 & 0 & 0 & 4 & 4 \\
\hline 2 & M & 66 & 2 & 2 & 4 & 2 & 6 & 8 \\
\hline 3 & M & 44 & 8 & 8 & 16 & 10 & 11 & 21 \\
\hline 4 & M & 59 & 2 & 0 & 2 & 4 & 5 & 9 \\
\hline 5 & M & 71 & 0 & 0 & 0 & 4 & 4 & 8 \\
\hline 6 & M & 74 & 3 & 2 & 5 & 3 & 5 & 8 \\
\hline 7 & $\mathrm{~F}$ & 65 & 2 & 7 & 9 & 4 & 10 & 14 \\
\hline 8 & M & 28 & 0 & 0 & 0 & 0 & 0 & 0 \\
\hline 9 & $\mathrm{~F}$ & 76 & 0 & 0 & 0 & 0 & 0 & 0 \\
\hline 10 & M & 70 & 4 & 8 & 12 & 5 & 11 & 16 \\
\hline 11 & $\mathrm{~F}$ & 57 & 2 & 5 & 7 & 2 & 6 & 8 \\
\hline 12 & F & 69 & 2 & 2 & 4 & 3 & 2 & 5 \\
\hline 13 & F & 63 & 2 & 0 & 2 & 2 & 5 & 7 \\
\hline 14 & $\mathrm{~F}$ & 47 & 1 & 2 & 3 & 4 & 3 & 7 \\
\hline 15 & M & 61 & 5 & 2 & 7 & 6 & 3 & 9 \\
\hline 16 & M & 67 & 0 & 0 & 0 & 5 & 8 & 13 \\
\hline 17 & M & 69 & 2 & 1 & 3 & 2 & 6 & 8 \\
\hline 18 & $\mathrm{~F}$ & 67 & 2 & 2 & 4 & 7 & 2 & 9 \\
\hline Total & & & 37 & 41 & $78^{\mathrm{a}}$ & $63^{\mathrm{b}}$ & 91 & 154 \\
\hline Median & & & 2 & 2 & 3.5 & 3.5 & 5 & 8 \\
\hline Range & & & $0-8$ & $0-8$ & $0-16$ & $0-10$ & $0-11$ & $0-21$ \\
\hline
\end{tabular}

Patients: M, male; F, female. The number of DNA copy number changes: G, gain; L, loss; T, total. ${ }^{\mathrm{a} p}<0.05 \mathrm{CGH}$ vs HR-CGH, ${ }^{\mathrm{b}} \mathrm{p}<0.05$ gains vs losses by HR-CGH.

parts. The nonparametric Wilcoxon rank-sum test was used for the variables with non-normal distributions, and the paired t-test was used for the variables with normal distributions. Differences of $\mathrm{p}<0.05$ were considered statistically significant. Each of the two chromosomal arms was counted individually in the total chromosomal aberrations summary. An aberration had to be identified in at least $15 \%$ of the cases in the central or peripheral tumour parts on order to be considered for the comparison between these two sections.

\section{Results}

Chromosomal aberrations detected by $C G H$ and HR-CGH. In order to make a comparison of CGH and HR-CGH sensitivity, samples from the central part of GMBs were chosen. A total of 78 different changes were observed using conventional CGH (0-16 per tumour, median 3.5) and 154 using HR-CGH $(0-21$ per tumour, median 8$)(\mathrm{p}<0.05)$ (Table I). The frequency of the losses and gains of chromosomal material was equivalent when using $\mathrm{CGH}$ (median 2 vs median 2), while the losses were more frequent than the gains when using HR-CGH (median 5 vs median 3.5) $(\mathrm{p}<0.05)$. There were five patients with no evidence of genetic changes using $\mathrm{CGH}$, while HR$\mathrm{CGH}$ was able to disclose changes for three of them (Table I).
The representation of the most frequent chromosomal changes revealed by both methods was similar: The loss of 10 and $9 p$, the gain of $7,3 q$ and 19 , and the amplification of 7 p12 and 12q13-q15. However, HR-CGH was always more effective in chromosomal change detection and it was also able to detect new aberrations that were only rarely detectable by CGH: The loss of $6,14 q, 15 q$ and $18 q$, and the gain of 19 (Fig. 1A and B).

Re-evaluation of HR-CGH. In order to re-evaluate the HRCGH results, I-FISH was performed in select cases, including when the $\mathrm{CGH}$ profiles did not exceed the given thresholds while HR-CGH showed a gain or loss of chromosomal sections or of the whole chromosome. In each case, the chromosome that showed a normal HR-CGH profile was chosen as the reference chromosome and was co-hybridized with centromeric or locus-specific probes for the re-evaluation of chromosomes or chromosomal loci (Table II). Using this approach, the clonal proportion of chromosomal changes detectable by HR-CGH was established to be $\sim 20 \%$.

Chromosomal aberrations in central and peripheral parts of $G B M$. The spectrum and frequency of chromosomal changes detected by HR-CGH in the central and peripheral sections of 


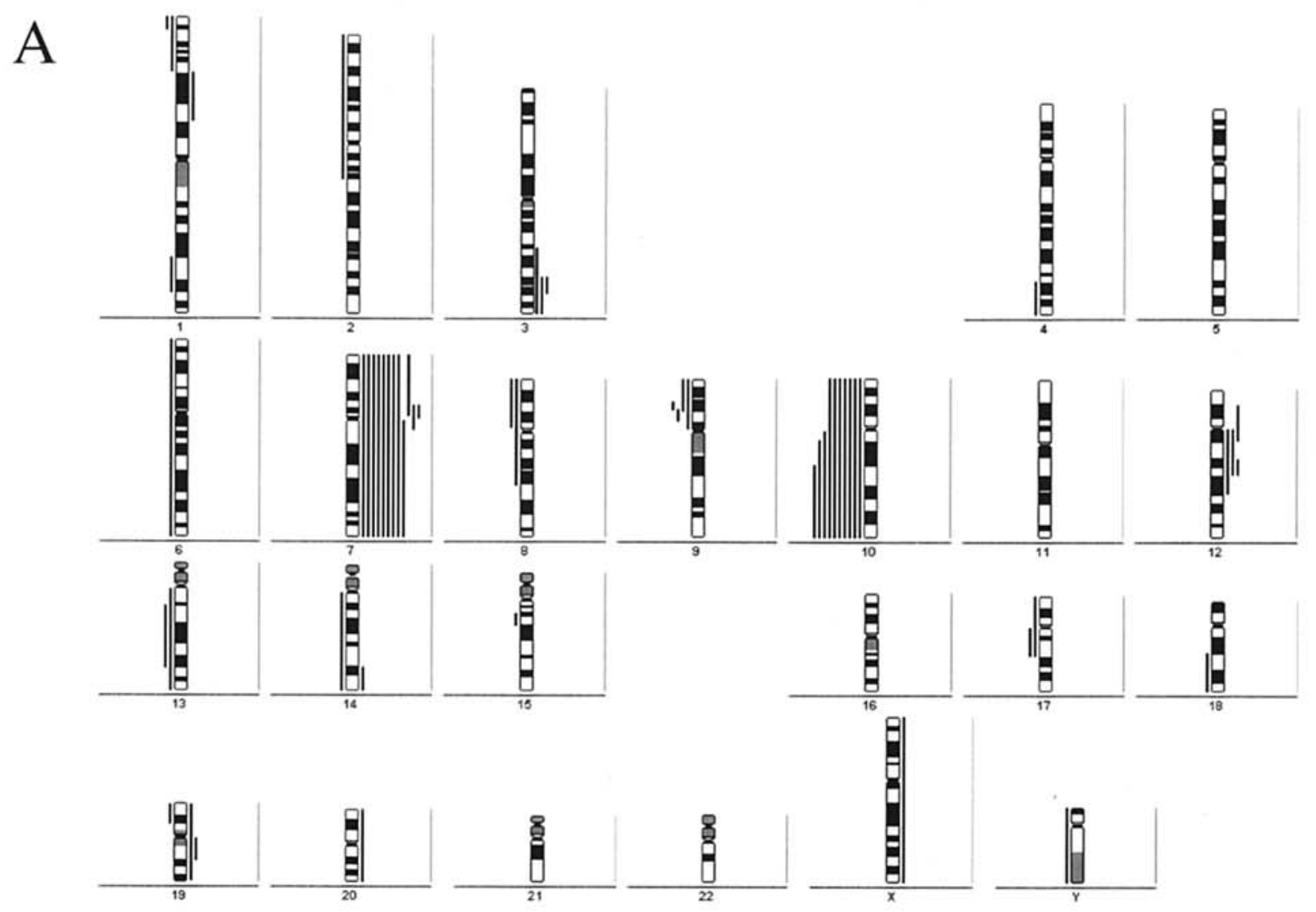

\section{B}

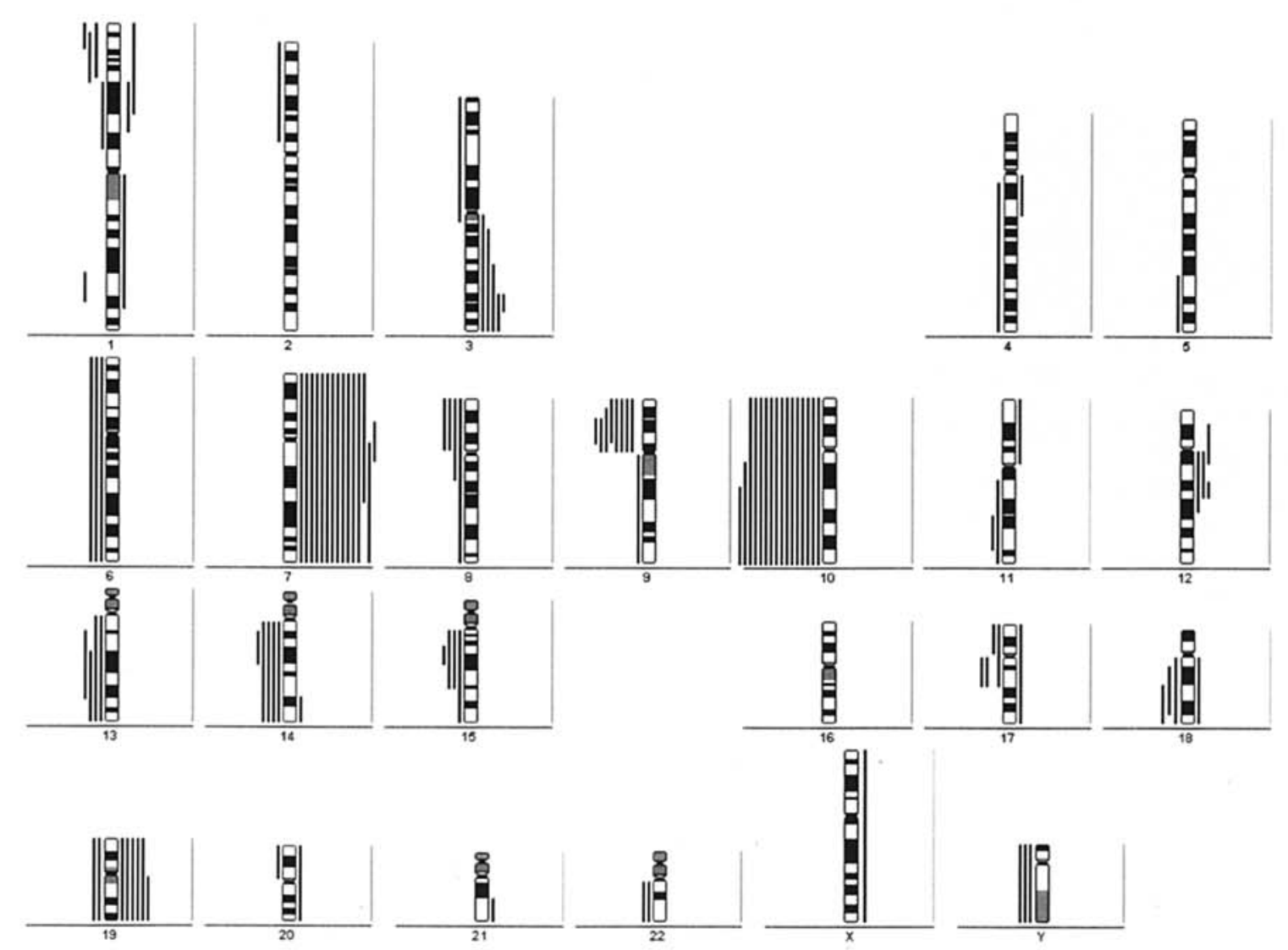

Figure 1. A summary of the copy number alternations in the tumour samples from 18 patients with GBM analysed by CGH (A) or HR-CGH (B). The vertical lines on the left of each chromosome ideogram represent losses, and on the right correspond to gains. 
Table II. HR-CGH re-evaluations performed on fixed tumour touch imprints by the I-FISH technique.

\begin{tabular}{|c|c|c|c|}
\hline $\begin{array}{l}\text { HR-CGH } \\
\text { change }\end{array}$ & Chromosome probe & $\begin{array}{l}\text { Locus of } \\
\text { interest }\end{array}$ & $\begin{array}{c}\text { I-FISH } \\
\% \text { (number of signals) }\end{array}$ \\
\hline$-1 p 36$ & Vysis LSI 1p36 SO/1q25 SG Probe & $1 \mathrm{p} 36$ & $18(2 x \mathrm{SG}, 1 \mathrm{x} \mathrm{SO})$ \\
\hline$-2 \mathrm{p}$ & Vysis LSI ALK Dual Color Probe & $2 \mathrm{p} 23$ & $36(1 \mathrm{x})$ \\
\hline+7 & Aquarius ${ }^{\circledR}$ Enumeration Probe Chromosome 7 (Green) & cep 7 & $27(3 x)$ \\
\hline+7 & Aquarius ${ }^{\circledR}$ Enumeration Probe Chromosome 7 (Green) & cep 7 & $33(3 x)$ \\
\hline$-9 q$ & Vysis LSI abl SO/bcr SG & $9 q 34$ & $53(2 \times \mathrm{SG}, 1 \times \mathrm{SO})$ \\
\hline-10 & Aquarius ${ }^{\circledR}$ Enumeration Probe Chromosome 10 (Red) & cep 10 & $30(1 \mathrm{x})$ \\
\hline-10 & Aquarius ${ }^{\circledR}$ Enumeration Probe Chromosome 10 (Red) & cep 10 & $31(1 \mathrm{x})$ \\
\hline$-14 q$ & Vysis LSI IgH SG/MYC SO/CEP 8 SA Probe & $14 q 32.3$ & 17 (2x SA, 1x SG) \\
\hline$-17 q 11.2-12$ & Vysis Her2/Neu SO/CEP 17 SG & $17 q 11.2-12$ & $50(2 x \mathrm{SG}, 1 \times \mathrm{SO})$ \\
\hline+17 & $\begin{array}{l}\text { Q-BIOgene } 17 \text { p13 specific probe (Green)/17 } \\
\text { aSatellite Probe (Red) }\end{array}$ & cep 17 & $26(3 x \mathrm{SG}, 3 x \mathrm{SO})$ \\
\hline$-19 q$ & Vysis LSI 19q13 SO/19p13 SG Probe & $19 q 13$ & $53(2 x \mathrm{SG}, 1 \times \mathrm{SO})$ \\
\hline+19 & Vysis LSI 19q13 SO/19p13 SG Probe & $19 \mathrm{p} 13,19 \mathrm{q} 13$ & $52(3 x$ SG, $3 x$ SO $)$ \\
\hline
\end{tabular}

HR-CGH was able to detect clones with a prevalence of $17-50 \%$, that were not detectable by CGH. Fluorophores: SO, Spectrum Orange ${ }^{\mathrm{TM}}$; SG, Spectrum Green ${ }^{\mathrm{TM}}$; SA, Spectrum Aqua ${ }^{\mathrm{TM}}$.

Table III. A summary of the DNA copy number changes detected by HR-CGH in the central and peripheral tumour parts in the samples taken from 18 patients with GBM.

\begin{tabular}{lrrrrrrr}
\hline & \multicolumn{3}{c}{ Central part } & & \multicolumn{3}{c}{ Peripheral part } \\
\cline { 2 - 4 } \cline { 7 - 8 } & G & L & T & & G & L & T \\
\hline Total & $63^{\mathrm{a}}$ & 91 & 154 & & $71^{\mathrm{a}}$ & 88 & 159 \\
Median & 3.5 & 5 & 8 & & 3.5 & 5 & 9 \\
Range & $0-10$ & $0-11$ & $0-21$ & & $0-11$ & $0-10$ & $0-21$ \\
\hline
\end{tabular}

The number of DNA copy number changes: G, gain; L, loss; T, total. ${ }^{\mathrm{a}} \mathrm{p}<0.05$ gains vs losses.

GBM were almost equivalent. A total of 154 different changes were observed in the central parts $(0-21$ per tumour, median 8 ) and 159 in the peripheral parts (0-21 per tumour, median 9) (Table III). The losses of chromosomal material were more frequent than the gains in both tumour parts $(\mathrm{p}<0.05)$. The most frequent losses were detected at chromosomes 10 , $9 \mathrm{p}$ and $14 \mathrm{q}$, while the most frequent gains occurred at chromosomes 7, 19 and 3q (Table IV). Amplifications were frequent at loci 7 p12 and 12q13-q15 in both tumour parts.

In general, 22 aberrations were present in the central tumour parts in at least $15 \%$ of the tumours, whereas three of these aberrations (the loss of 8p, 13q14, and 18q12-q21) occurred at a lower frequency in the peripheral parts (Table IV). However, these differences were not statistically significant. Therefore, no genetic change was predominantly associated with either one of the tumour parts.
Table IV. A comparison of the common aberrations revealed by HR-CGH in the central and peripheral parts of the tumour samples from 18 patients with GBM.

\begin{tabular}{|c|c|c|}
\hline \multirow[t]{2}{*}{$\begin{array}{l}\text { Abnormality } \\
\text { (minimal region) }\end{array}$} & \multicolumn{2}{|c|}{$\begin{array}{l}\text { Total number }(\%) \text { of samples } \\
\text { with the respective change }\end{array}$} \\
\hline & Central part & Peripheral part \\
\hline \multicolumn{3}{|l|}{ Losses } \\
\hline 1p (1p36.1) & $3 / 18(17)$ & 4/18 (22) \\
\hline $6 \mathrm{p}$ & $3 / 18$ (17) & $3 / 18$ (17) \\
\hline $6 q$ & 3/18 (17) & 3/18 (17) \\
\hline $8 p$ & $4 / 18(22)$ & $2 / 18(11)$ \\
\hline $9 p(9 p 21)$ & 8/18 (44) & $10 / 18(56)$ \\
\hline $10 p$ & 13/18 (72) & $12 / 18(67)$ \\
\hline $10 q(10 q 22$-qter) & $15 / 18(83)$ & $14 / 18(78)$ \\
\hline $13 q(13 q 21-q 31)$ & $4 / 18(22)$ & $5 / 18(28)$ \\
\hline $14 q(14 q 12-q 21)$ & $5 / 18(28)$ & 4/18 (22) \\
\hline $15 q(15 q 15-q 21)$ & $4 / 18(22)$ & $4 / 18(22)$ \\
\hline $17 q(17 q 11-q 21)$ & $3 / 18(17)$ & $3 / 18(17)$ \\
\hline $18 q(18 q 12-21)$ & $3 / 18(17)$ & $2 / 18(11)$ \\
\hline $\mathrm{Y}$ & 3/18 (17) & 4/18 (22) \\
\hline \multicolumn{3}{|l|}{ Gains } \\
\hline $3 q(3 q 26.1-26.3)$ & $5 / 18(28)$ & 4/18 (22) \\
\hline $7 \mathrm{p}$ & $14 / 18(78)$ & $14 / 18(78)$ \\
\hline 7q (7q11.1-q22) & $15 / 18(83)$ & $14 / 18(78)$ \\
\hline $19 p$ & $5 / 18(28)$ & 6/18 (33) \\
\hline $19 q$ & 6/18 (33) & 6/18 (33) \\
\hline \multicolumn{3}{|l|}{ Amplifications } \\
\hline $7 \mathrm{p} 12$ & 4/18 (22) & 4/18 (22) \\
\hline $12 q 13-q 15$ & 3/18 (17) & 3/18 (17) \\
\hline
\end{tabular}




\section{Discussion}

This study was designed to compare conventional CGH with the newly developed HR-CGH technique in order to ascertain the potential of HR-CGH in aiding the routine classification of GBM tumours. A series of 18 histologically characterized GBM tumours was examined using these two methods to compare their detection sensitivity, as well the spectrum of chromosomal changes in the central and peripheral parts of these tumours.

The detection limit of HR-CGH was established at 4-5 Mb, with an aberrant clone prevalence of at least $20 \%$ (Table II). HR-CGH detected clones with a prevalence of $20-50 \%$ which were not detectable by $\mathrm{CGH}$, and thus was able to reveal nearly $50 \%$ more aberrations than CGH. This increment was especially prominent in the number of losses. Similar results, i.e. more frequent losses than gains, were also observed in another CGH study (22). One possible explanation is that duplications are more difficult to detect than deletions. The green:red ratio for duplications is $3: 2$, while the one for deletions is $1: 2(23)$. This is a very important fact to realize especially in the case of small aberrations and/or aberrations with lower prevalence detection. Even three apparently negative patients were shown to possess some genetic alterations by using HR-CGH instead of CGH.

Our study supported the trend of prominent chromosomal abnormalities in GBM described in other reports. $(3,5,6,9,13,19,22,24-28)$. Chromosomal changes most often involved chromosomes 7, 10,9p, 19, 3q and 13q, which are important indicators of GBM progression. As documented in Fig. $1 \mathrm{~A}$ and $\mathrm{B}$, these prominent changes were identified using both CGH and HR-CGH methods, although HR-CGH demonstrated a higher prevalence. The HR-CGH representation of the chromosomal abnormalities was comparable not only with previous $\mathrm{CGH}$ studies including a similar number of patients examined but also with studies that involved a higher number of patients $(3,13,25)$.

HR-CGH revealed two times more alterations of $9 \mathrm{p}$ loci than conventional CGH (Fig. 1A and B). Tumour suppressor genes (TSGs) that play important roles in cell cycle regulation were shown to be located in this region (29).

Losses in chromosome $13 \mathrm{q}$ have been reported previously in studies of high-grade gliomas $(25,26,28)$, possibly implicating the inactivation of the retinoblastoma gene at $13 q 14$. A commonly deleted region in our study was $13 q 21-$ q31, which was also found to be lost in the study of Mohapatra et al (3). Possible TSGs localized in this region could also be associated with tumour progression.

There are certain TSGs on $19 \mathrm{q}$ that are frequently involved in the progression from low-grade astrocytoma to secondary glioblastoma (30). We detected the loss of chromosome 19 in only a few cases, whereas there were frequent gains in both arms of chromosome 19 (Fig. 1B). Similar results were observed by another team in 72 primary GBMs (3). Gains of chromosome 19 were detected mostly by HR-CGH, which is possibly due to the fact that these abnormalities occurred at a lower frequency. However, the presence of this chromosome could have a significant impact on patient treatment. Huhn et al (9) has shown that the simultaneous gain of chromosomes 7 and 19 was found in
$30 \%$ of the radiation-resistant cases but was absent in the radiation-sensitive group.

Nevertheless, there were some changes that were observed predominantly or only with HR-CGH, and their impact on the survival of patients with GBM has also been documented (7). One of these changes is the invariable finding of a partial or complete loss of $14 \mathrm{q}$. This loss has been described in oligodendrogliomas. However, alterations of this region are not often described in GBM studies. Kros et al (21) reported the loss of the entire chromosome $14 \mathrm{q}$ in the high-grade tumour regions of a large tumour resection specimen. Using high-resolution genome-wide allelotype analysis, two common regions of deletion on chromosome 14 were mapped to 14q22.3-q32.1 and 14q32.1-qter (31). However, we found $14 \mathrm{q} 12-\mathrm{q} 21$ as a commonly deleted region, so there could be more regions whose deletion plays an important role in the pathogenesis of GBM.

The loss of the whole of chromosome 6 is another alteration detected primarily by HR-CGH. Several studies have described the potential role of the $6 \mathrm{q}$ deletion in malignant gliomas $(32,33)$. Weber et al (34) found that the $6 \mathrm{q}$ loss was more common in the shorter progression-free interval group. Ichimura et al (35) confirmed the high frequency of chromosome 6 deletions in anaplastic astrocytomas and GBM using array-CGH, and identified two novel commonly deleted regions that could harbour TSGs. Previous studies have also shown that the loss of chromosomes 6 and 14 occurs more frequently in recurrent than in primary tumours. This suggests that these changes could play a role in GBM recurrence and/or the development of resistance to therapy (24).

Other affected chromosomal regions were $8 p, 15 q$ and 18q (Fig. 1B). Some studies, in accordance with our results, revealed the loss of the whole or part of the long arm of chromosome $15(3,21)$, while others report gains and even the amplification of some regions of this chromosome $(36,37)$. The prognostic effects of these alterations remain unreported in literature.

The frequent loss of $18 \mathrm{q}$ in the context of gliomas was described in 22 glioma-derived cell lines using a custommade CGH array (38). Wooten et al (32) described the loss of the long arm of chromosome 18 and the short arm of chromosome 8 as the most frequently deleted loci specific to GBM, which can either lead to de novo GBM or permit existing astrocytomas to progress to GBM. The frequent loss of chromosome 8p, observed in our study by HR-CGH, has not yet been described in $\mathrm{CGH}$ studies of gliomas.

The lesser-known chromosome alterations we report here can be useful for higher-resolution mapping studies to precisely identify novel genes which could serve as targets for the future treatment of this malignancy.

The term 'multiforme' in GBM indicates the histological heterogeneity, and within a single tumour, areas with lowand high-grade histological features can be visible. Intratumoural genetic heterogeneity has been detected using CGH on microdissected glioblastoma tumour cells $(19,21)$, and a large number of novel genes with distinct expression patterns in high- and low-grade gliomas have been identified (39). In contrast, Walker et al (40) found identical genetic losses in all areas of histological differentiation in 13 glioma tumours with intratumoural heterogeneity. We identified certain 
differences in the numbers and representation of chromosomal aberrations between the central and peripheral parts of single tumours using newly developed HR-CGH. In our study, the comparison of the genetic change distribution in both areas showed that there are minimal differences in the representation of common genetic changes (Table III). Although aberrations of chromosomes 11 and 16 were observed in the peripheral but not the central tumour parts, this fact had no statistical significance. This contrast with other CGH studies could be due to the fact that our examined tumour parts were not histologically graded. They differed only in topological localization and therefore both parts could conceivably contain both low- and high-grade areas.

We have confirmed that GBM harbours multiple genetic abnormalities and that DNA isolated from single tumours varies widely in the number and location of chromosomal changes observed. However, genetic heterogeneity did not exist within the same tumour if compared localities differed only topologically, and not histologically. The molecular classification of gliomas is becoming increasingly important clinically as an adjunct to histopathological diagnosis. Our results have shown that $\mathrm{HR}-\mathrm{CGH}$ is a comprehensive and rapid methodological approach to the analysis of global and locus-specific genomic alterations in glial tumours. Therefore, it could serve as a molecular tool for improved diagnostics and therapeutic decision-making in these tumours.

\section{Acknowledgements}

This study was supported by grants COST OC B19.001 and VZ MSM 0021622415. We thank Mrs. Iva Slamova for her technical assistance.

\section{References}

1. Kleihues P, Burger PC and Scheuthauer BW: The new WHO classification of brain tumours. Brain Pathol 3: 255-268, 1993.

2. Benjamin R, Capparella J and Brown A: Classification of glioblastoma multiforme in adults by molecular genetics. Cancer J Sci Am 9: 82-90, 2003.

3. Mohapatra G, Bollen AW, Kim DH, Lamborn K, Moore DH, Prados MD and Feuerstein BG: Genetic analysis of glioblastoma multiforme provides evidence for subgroups within the grade. Genes Chromosomes Cancer 21: 195-206, 1998.

4. Smith SJ, Tachibana I, Passe SM, Huntley BK, Borell TJ, Iturria N, O'Fallon JR, Schaefer PL, Scheithauer BW, James CD, Buckner JC and Jenkins RB: PTEN mutation, EGFR amplification and outcome in patients with anaplastic astrocytoma and glioblastoma multiforme. J Natl Cancer Inst 93: 1246-1256, 2001.

5. Bigner SH, Matthews MR, Rasheed BK, Wiltshire RN, Friedman HS, Friedman AH, Stenzel TT, Dawes DM, McLendon RE and Bigner DD: Molecular genetic aspects of oligodendrogliomas including analysis by comparative genomic hybridization. Am J Pathol 155: 375-386, 1999.

6. Squire JA, Arab S, Marrano P, Bayani J, Karaskova T, Taylor M, Becker L, Rutka J and Zielenska M: Molecular cytogenetic analysis of glial tumours using spectral karyotyping and comparative genomic hybridization. Mol Diagn 6: 93-108, 2001.

7. Burton EC, Lamborn KR, Feuerstein BG, Prados M, Scott J, Forsyth P, Passe S, Jenkins RB and Aldape KD: Genetic aberrations defined by comparative genomic hybridization distinguish long-term from typical survivors of glioblastoma. Cancer Res 62: 6205-6210, 2002.

8. Batchelor TT, Betensky RA, Esposito JM, Pham LD, Dorfman MV, Piscatelli N, Jhung S, Rhee D and Louis DN: Age dependent prognostic effects of genetic alterations in glioblastoma. Clin Cancer Res 10: 228-233, 2004.
9. Huhn SL, Mohapatra G, Bollen A, Lamborn K, Prados MD and Feuerstein BG: Chromosomal abnormalities in glioblastoma multiforme by comparative genomic hybridization: correlation with radiation treatment outcome. Clin Cancer Res 5: 1435-1443, 1999.

10. Wemmert S, Ketter R, Rahnenfuhrer J, Beerenwinkel N, Strowitzki M, Feiden W, Hartmann C, Lengauer T, Stockhammer F, Zang KD, Meese E, Steudel WI, von Diemling A and Urbschat S: Patients with high-grade gliomas harboring deletions of chromosomes $9 \mathrm{p}$ and $10 \mathrm{q}$ benefit from temozolomide treatment. Neoplasia 7: 883-893, 2005.

11. Kallioniemi A, Kallioniemi OP, Sudar D, Rutovitz D, Gray JW, Waldman F and Pinkel D: Comparative genomic hybridization for molecular cytogenetic analysis of solid tumours. Science 258: 818-821, 1992

12. Mao X and Hamoudi RA: Molecular and cytogenetic analysis of glioblastoma multiforme. Cancer Genet Cytogenet 122: 87-92, 2000.

13. Wiltshire RN, Rasheed BK, Friedman HS, Friedman AH and Bigner SH: Comparative genetic paterns of glioblastoma multiforme: Potential diagnostic tool for tumour classification. Neurooncology 2: 164-173, 2000.

14. Kirchhoff M, Gerdes T, Rose H, Maahr J, Ottesen AM and Lundsteen C: Detection of chromosomal gains and losses in comparative genomic hybridization analysis based on standard reference intervals. Cytometry 31: 163-173, 1998.

15. Kirchhoff M, Gerdes T, Maahr J, Rose H, Bentz M, Dohner H and Lundsteen C: Deletions below 10 megabasepairs are detected in comparative genomic hybridization by standard reference intervals. Genes Chromosomes Cancer 25: 410-413, 1999.

16. Kirchhoff T, Rose H, Maahr J, Gerdes T, Bugge M, Tommerup N, Tumer Z, Lespinasse J, Jensen PK, Wirth J and Lundsteen C: High resolution comparative genomic hybridization analysis reveals imbalances in dyschromosomal patients with normal or apparently balanced conventional karyotypes. Eur J Hum Genet 8: 661-668, 2000

17. Ness GO, Lybaek H and Houge G: Usefulness of high-resolution comparative genomic hybridization $(\mathrm{CGH})$, for detecting and characterizing constitutional chromosome abnormalities. Am J Med Genet 113: 125-136, 2002.

18. Kristensen TD, Wesenberg F, Jonsson OG, Carlsen NT, Forestier E, Kirchhoff M, Lundsteen C and Schimegelow K: High-resolution comparative genomic hybridization yields a high detection rate of chromosomal aberrations in childhood acute lymphoblastic leukaemia. Eur J Haematol 70: 363-372, 2003.

19. Jung V, Romeike BF, Henn W, Feiden W, Moringlane JR, Zang KD and Urbschat S: Evidence of focal genetic microheterogeneity in glioblastoma multiforme by area-specific CGH on microdissected tumor cells. J Neuropathol Exp Neurol 58: 993-999, 1999.

20. Loeper S, Romeike BF, Heckmann N, Jung V, Henn W, Feiden W, Zang KD and Urbschat S: Frequent mitotic errors in tumor cells of genetically micro-heterogeneous glioblastomas. Cytogenet Cell Genet 94: 1-8, 2001.

21. Kros JM, van Run PR, Alers JC, Avezaat CJ, Luider TM and van Dekken H: Spatial variability of genomic aberrations in large glioblastoma resection specimen. Acta Neuropathol 102: 103-109, 2001.

22. Maruno M, Yoshimine T, Muhammad AK, Ninomiya H, Kato A and Hayakawa T: Chromosomal aberrations detected by comparative genomic hybridization $(\mathrm{CGH})$ in human astrocytic tumours. Cancer Lett 135: 61-66, 1999.

23. Schoumans J, Nielsen K, Jeppesen I, Anderlid BM, Blennow E, Brondum-Nielesen $\mathrm{K}$ and Nordenskjold $\mathrm{M}$ : A comparison of different metaphase CGH methods for the detection of cryptic chromosome aberrations of defined size. Eur J Hum Genet 12: 447-454, 2004.

24. Kim DH, Mohapatra G, Bollen A, Waldman FM and Feuerstein BG: Chromosomal abnormalities in glioblastoma multiforme tumors and glioma cell lines detected by comparative genomic hybridization. Int J Cancer 60: 812-819, 1995.

25. Schlegel J, Scherthan H, Arens N, Stumm G and Kiessling M: Detection of complex genetic alterations in human glioblastoma multiforme using comparative genomic hybridization. J Neuropathol Exp Neurol 55: 81-87, 1996.

26. Weber RG, Sabel M, Reifenberger J, Sommer C, Oberstrass J, Reifenberger G, Kiessling M and Cremer T: Characterization of genomic alterations associated with glioma progression by comparative genomic hybridization. Oncogene 13: 983-994, 1996. 
27. Hui AB, Lo KW, Yin XL, Poon WS and Ng HK: Detection of multiple gene amplification in glioblastoma multiforme using array-based comparative genomic hybridization. Lab Invest 81: 717-723, 2001

28. Inda MM, Fan X, Munoz J, Perot C, Fauvet D, Danglot G, Palacio A, Madero P, Zazpe I, Portillo E, Tunon T, MartinezPenuela JM, Alfaro J, Eiras J, Bernheim A and Castresana JS: Chromosomal abnormalities in human glioblastomas: gain in chromosome $7 \mathrm{p}$ correlating with loss in chromosome 10q. Mol Carcinog 36: 6-14, 2003.

29. Collins VP: Progression as exemplified by human astrocytic tumours. Semin Cancer Biol 9: 267-276, 1999.

30. Nakamura M, Yang F, Fujisawa H, Yonekawa Y, Kleihues P and Ohgaki H: Loss of heterozygosity on chromosome 19 in secondary glioblastomas. J Neuropathol Exp Neurol 59: 539-543, 2000.

31. Hu J, Jiang C, Ng HK, Pang JC and Tong CY: Chromosome $14 \mathrm{q}$ may harbor multiple tumor suppressor genes in primary glioblastoma multiforme. Chin Med J 115: 1201-1204, 2002.

32. Wooten EC, Fults D, Duggirala R, Williams K, Kyritsis AP, Bondy ML, Levin VA and O'Connell P: A study of loss of heterozygosity at 70 loci in anaplastic astrocytoma and glioblastoma multiforme with implications for tumour evolution. Neurooncology 1: 169-176, 1999.

33. Miyakawa A, Ichimura K, Schmidt EE, Varmeh-Ziaie S and Collins VP: Multiple deleted regions on the long arm of chromosome 6 in astrocytic tumours. Br J Cancer 82: 543-549, 2000.

34. Weber RG, Sommer C, Albert FK, Kiessling M and Cremer T: Clinically distinct subgroups of glioblastoma multiforme studied by comparative genomic hybridization. Lab Invest 74 : 108-119, 1996.
35. Ichimura K, Mungall AJ, Fiegler H, Pearson DM, Dunham I, Carter NP and Collins VP: Small regions of overlapping deletions on $6 \mathrm{q} 26$ in human astrocytic tumours identified using chromosome 6 tile path array-CGH. Oncogene 25: 1261-1271, 2006.

36. Brunner C, Jung V, Henn W, Zang KD and Urbschat S: Comparative genomic hybridization reveals recurrent enhancements on chromosome 20 and in one case combined amplification sites on $15 \mathrm{q} 24 \mathrm{q} 26$ and $20 \mathrm{p} 11 \mathrm{p} 12$ in glioblastomas. Cancer Genet Cytogenet 121:124-127, 2000.

37. Ramirez T, Thoma K, Taja-Chayeb L, Efferth T, Herrera LA, Halatsch ME and Gebhart E: Specific pattern of DNA copy numer gains and losses in eight new glioblastoma multiforme cell lines. Int J Oncol 23: 453-460, 2003.

38. Saigusa K, Hashimoto N, Tsuda H, Yokoi S, Maruno M, Yoshimine T, Aoyagi M, Ohno K, Imoto I and Inazawa J: Overexpressed Skp2 within 5p amplification detected by arraybased comparative genomic hybridization is associated with poor prognosis of glioblastomas. Cancer Sci 96: 676-683, 2005 .

39. Rickman DS, Bobek MP, Misek DE, Kuick R, Blaivas M, Kurnit DM, Taylor J and Hanash SM: Distinctive molecular profiles of high-grade and low-grade gliomas based on oligonucleotide microarray analysis. Cancer Res 61: 6885-6891, 2001.

40. Walker C, du Plessis DG, Joyce KA, Machell Y, ThomsonHehir J, Al Haddad SA, Broome JC and Warnke PC: Phenotype versus genotype in gliomas displaying inter- or intratumoral histological heterogeneity. Clin Cancer Res 9: 4841-4851, 2003. 